\title{
Pharmacytes: An Ideal Vehicle for Targeted Drug Delivery
}

\author{
Robert A. Freitas, Jr. \\ Institute for Molecular Manufacturing, 555 Bryant Street, Suite 354, Palo Alto, California 94301, USA
}

\begin{abstract}
An ideal nanotechnology-based drug delivery system is a pharmacyte-a self-powered, computercontrolled medical nanorobot system capable of digitally precise transport, timing, and targeted delivery of pharmaceutical agents to specific cellular and intracellular destinations within the human body. Pharmacytes may be constructed using future molecular manufacturing technologies such as diamond mechanosynthesis which are currently being investigated theoretically using quantum $a b$ initio and density-functional computational methods. Pharmacytes will have many applications in nanomedicine such as initiation of apoptosis in cancer cells and direct control of cell signaling processes.
\end{abstract}

Keywords: Apoptosis, Cell Signaling, Drug Delivery, Microbivore, Microrobot, Nanomedicine, Nanomotor, Nanorobot, Nanotechnology, Pharmacyte, Respirocyte.

\section{INTRODUCTION}

What would an ideal drug delivery vehicle look like? To start with, it would be targetable not just to specific tissues or organs, but to individual cellular addresses within a tissue or organ. Alternatively, it would be targetable to all individual cells within a given tissue or organ that possessed a particular characteristic (e.g., all cancer cells, or all bacterial cells of a definite species, etc.). This ideal vehicle would be biocompatible and virtually $100 \%$ reliable, with all drug molecules being delivered only to the desired target cells and none being delivered elsewhere so that unwanted side effects are eliminated. The ideal vehicle would remain under the continuous control of the supervising physician, including post-administration. Even after the vehicles had been injected into the body, the doctor would still be able to activate or inactivate them remotely, or alter their mode of action or operational parameters. Once treatment was completed, all of the vehicles could be removed intact from the body, leaving no trace of their presence. Let's call this hypothetical ideal drug delivery vehicle a "pharmacyte."

The application of advanced nanotechnology to medicine, or nanomedicine ${ }^{1-3}$-in particular, the future engineering discipline of medical nanorobotics-will eventually make possible the design, fabrication, and therapeutic deployment of pharmacytes. Pharmacytes will be self-powered, computer-controlled nanorobotic systems

\footnotetext{
*Author to whom correspondence should be addressed.
}

capable of digitally precise transport, timing, and targeted delivery of pharmaceutical agents to specific cellular and intracellular destinations within the human body. Drug molecules could be purposely delivered to one cell, but not to an adjacent cell, in the same tissue. To fully appreciate the scope of this future development it is helpful to briefly review some of the background and recent history of medical nanorobotics.

\section{FROM NANOPARTICLES TO NANOROBOTS}

To bridge the gap in our knowledge between present-day nanoparticle-based technologies and future nanorobotic technologies, a great deal of research remains to be done. In the relatively near term, over the next 5 years, prenanorobotic nanomedicine can address many important medical problems by using nanoscale-structured materials and basic nanodevices for drug delivery that can already be manufactured today-most notably organic polymer or lipid-based systems such as polymeric micelles, liposomes and solid lipid nanoparticles, and various nanocrystalbased systems, many of which have already advanced to marketed products. Surveys of these technologies are available elsewhere, ${ }^{2,3}$ so here we consider just a few selected examples of nanoparticle-related work that may exemplify early steps toward the more sophisticated capabilities that nanorobots will ultimately possess.

Kopelman's group at the University of Michigan has developed dye-tagged nanoparticles to be inserted into 
living cells as biosensors. This quickly led to more complex nanoparticle platforms incorporating a variety of plug-in modules, creating molecular nanodevices for the early detection and therapy of brain cancer. ${ }^{4}$ In this instance, one type of nanoparticle is attached to a cancer cell antibody that adheres to cancer cells, but is also affixed with a contrast agent to make the particle highly visible during MRI while also enhancing the selective cancerkilling effect during subsequent laser irradiation of the treated brain tissue.

Baker's group at the University of Michigan works with dendrimers, tree-shaped synthetic molecules with a regular branching structure emanating outward from a core. The outermost layer can be functionalized with other useful molecules such as genetic therapy agents, decoys for viruses, or anti-HIV agents. The next step is to create dendrimer cluster agents, multi-component nanodevices called tecto-dendrimers built up from a number of single-dendrimer modules. ${ }^{5,6}$ These modules may perform specialized functions such as diseased cell recognition, diagnosis of disease state, therapeutic drug delivery, location reporting, and therapy outcome reporting. The framework can be customized to fight a particular cancer simply by substituting any one of many possible distinct cancer recognition or "targeting" dendrimers. The larger trend in medical nanomaterials is to migrate from single-function molecules to multilayer or multimodule entities that can do many things but only at certain times, or under certain conditions, or in a particular sequence. This exemplifies a continuing and inevitable technological evolution toward a device-oriented nanomedicine, working from the bottom up.

On the top-down pathway, there are ongoing attempts to build microrobots for in vivo medical use. In 2002, Ishiyama et al. at Tohoku University developed tiny magnetically-driven spinning screws intended to swim along veins and carry drugs to infected tissues or even to burrow into tumors and kill them with heat. ${ }^{7}$ In 2003, the "MR-Sub" project of Martel's group at the NanoRobotics Laboratory of Ecole Polytechnique in Montreal tested using variable MRI magnetic fields to generate forces on an untethered microrobot containing ferromagnetic particles, developing sufficient propulsive power to direct the small device through the human body. ${ }^{8}$ Brad Nelson's team at the Swiss Federal Institute of Technology in Zurich has continued this approach. In 2005 they reported ${ }^{9}$ the fabrication of a microscopic robot small enough $(\sim 200 \mu \mathrm{m})$ to be injected into the body through a syringe. They hope this device or its descendants might someday be used to deliver drugs or perform minimally invasive eye surgery. Nelson's simple microrobot has successfully maneuvered through a watery maze using external energy from magnetic fields, with different frequencies able to vibrate different mechanical parts on the device to maintain selective control of various functions. Gordon's group at the University of Manitoba has also proposed magnetically-controlled "cytobots" and "karyobots" for performing wireless intracellular and intranuclear surgery. ${ }^{10}$ These approaches illustrate the first steps toward developing the ability to externally control microscopic objects after they have been placed inside the human body, an important capability for future medical nanorobots.

Other methods for controlling the activity of the tiniest robotic devices-or even individual macromoleculesare being investigated in the laboratory. Most interestingly, Jacobson and colleagues ${ }^{11}$ have attached tiny radio-frequency antennas- 1.4 nanometer gold nanocrystals of less than 100 atoms-to DNA. When a $\sim 1$ $\mathrm{GHz}$ radio-frequency magnetic field is transmitted into the tiny antennas, alternating eddy currents induced in the nanocrystals produce highly localized inductive heating, causing the double-stranded DNA to separate into two strands in a matter of seconds in a fully reversible dehybridization process that leaves neighboring molecules untouched. The long-term goal is to apply the antennas to living systems and control DNA (e.g., gene expression, giving the ability to turn genes on or off) via remote electronic switching. Such a tool could give pharmaceutical researchers a way to simulate the effects of potential drugs, which also turn genes on and off. The gold nanocrystals can be attached to proteins as well as DNA, opening up the possibility of future radio frequency biology electronically controlling more complex biological processes such as enzymatic activity, protein folding and biomolecular assembly. ${ }^{12}$

Motors and bearings for nanoscale machines have received a great deal of experimental attention, including the 78-atom chemically-powered rotating nanomotor synthesized in 1999 by Kelly, ${ }^{13}$ a chemically-powered rotaxane-based linear motor exerting $\sim 100 \mathrm{pN}$ of force with a $1.9 \mathrm{~nm}$ throw and a $\sim 250 \mathrm{sec}$ contraction cycle by Stoddart's group, ${ }^{14} \mathrm{a}$ UV-driven catenane-based ring motor by Wong and Leigh, ${ }^{15}$ and an artificial 58 -atom motor molecule that spins when illuminated by solar energy by Feringa. ${ }^{16}$ Zettl's group at U.C. Berkeley has experimentally demonstrated an essentially frictionless bearing made from two co-rotating nested nanotubes, ${ }^{17}$ which can also serve as a mechanical spring because the inner nanotube "piston" feels a restoring force as it is extracted from the outer nanotube "jacket". Zettl's group then fabricated a nanomotor mounted on two of these nanotube bearings, demonstrating the first electrically powered nanoscale motor. ${ }^{18}$

In 2005, Tour's group at Rice University reported ${ }^{19}$ constructing a tiny molecular "nanocar" measuring 3-4 nanometers across that consists of a chassis, two freely rotating axles made of well-defined rodlike acetylenic structures with a pivoting suspension, and wheels made of $\mathrm{C}_{60}$ buckyball molecules that can turn independently because the bond between them and the axle is freely rotatable. Placed on a gold surface at $170{ }^{\circ} \mathrm{C}$, the nanocar 
spontaneously rolls on all four wheels, but only along its long axis in a direction perpendicular to its axles (a symmetrical three-wheeled variant just spins in place). When pulled with an STM tip, the nanocar cannot be towed sideways - the wheels dig in, rather than rolling. A larger, more functionalized version of the nanocar might carry other molecules along and dump them at will. Indeed, the Rice team has apparently "already followed up the nanocar work by designing a light-driven nanocar and a nanotruck that's capable of carrying a payload." 20

The greatest power of nanomedicine will emerge, perhaps in the 2020s, when we can design and construct complete artificial medical nanorobots using rigid diamondoid nanometer-scale parts such as molecular gears and bearings. ${ }^{21}$ Complete artificial nanorobots may possess a full panoply of autonomous subsystems including onboard sensors, pumps, motors, manipulators, clocks, power supplies, communication systems, navigation systems, and molecular computers, as has been extensively reviewed elsewhere. ${ }^{1}$ But getting all of these nanoscale components to spontaneously self-assemble in the right sequence will prove increasingly difficult as machine structures become more intricate. Making complex nanorobotic systems requires manufacturing techniques that can build a molecular structure by what is called positional assembly. This will involve picking and placing molecular parts one by one, moving them along controlled trajectories much like the robot arms that manufacture cars on automobile assembly lines. The procedure is then repeated over and over with all the different parts until the final product, such as a medical nanorobot, is fully assembled.

The positional assembly of diamondoid structures, some almost atom by atom, using molecular feedstock has been examined theoretically ${ }^{21,22}$ via computational models of diamond mechanosynthesis (DMS) using ab initio and density functional methods. DMS is the controlled addition of carbon atoms to the growth surface of a diamond crystal lattice in a vacuum manufacturing environment. Covalent chemical bonds are formed one by one as the result of positionally constrained mechanical forces applied at the tip of a scanning probe microscope apparatus, following a programmed sequence. In this manner, nanoparts may be fabricated and then assembled into working machines. Mechanosynthesis using silicon atoms was first achieved experimentally in $2003 .{ }^{23}$ Carbon atoms should not be far behind. $^{24}$

To be practical, molecular manufacturing must also be able to assemble very large numbers of medical nanorobots very quickly. Approaches under consideration include using replicative manufacturing systems or massively parallel fabrication, employing large arrays of scanning probe tips all building similar diamondoid product structures in unison. ${ }^{25}$ For example, simple mechanical ciliary arrays consisting of 10,000 independent microactuators on a $1 \mathrm{~cm}^{2}$ chip have been made at the Cornell
National Nanofabrication Laboratory for microscale parts transport applications, and similarly at IBM for mechanical data storage applications. ${ }^{26}$ Active probe arrays of 10,000 independently-actuated microscope tips have been developed by Mirkin's group at Northwestern University for dip-pen nanolithography using DNA-based "ink". ${ }^{27}$ Almost any desired 2D shape can be drawn using 10 tips in concert. Another microcantilever array manufactured by Protiveris Corp. has millions of interdigitated cantilevers on a single chip. Martel's group has investigated using fleets of independently mobile wireless instrumented microrobot manipulators called NanoWalkers to collectively form a nanofactory system that might be used for positional manufacturing operations. ${ }^{28}$ Zyvex Corp. (www.zyvex.com) of Richardson TX has a \$25 million, five-year, National Institute of Standards and Technology (NIST) contract to develop prototype microscale assemblers using microelectromechanical systems. This research may eventually lead to prototype nanoscale assemblers using nanoelectromechanical systems (NEMS).

Several conceptual designs of medical nanorobots have been published. For instance, the first theoretical design study $^{29}$ of a complete medical nanorobot ever published in a peer-reviewed journal (in 1998) described a hypothetical artificial mechanical red blood cell or "respirocyte" made of 18 billion precisely arranged structural atoms. The respirocyte is a bloodborne spherical $1-\mu \mathrm{m}$ diamondoid 1000-atmosphere pressure vessel with reversible moleculeselective pumps powered by endogenous serum glucose. This nanorobot would deliver 236 times more oxygen to body tissues per unit volume than natural red cells and would manage carbonic acidity, controlled by gas concentration sensors and an onboard nanocomputer. A mere $5 \mathrm{cc}$ therapeutic dose of $50 \%$ respirocyte saline suspension containing 5 trillion nanorobots could exactly replace the gas carrying capacity of the patient's entire 5.4 liters of blood. The supervising physician can transmit control commands via ultrasound signals that are received by acoustic sensors on the nanorobot hull. Another conceptual design is the nanorobotic artificial phagocytes called "microbivores" 30 that could patrol the bloodstream, seeking out unwanted pathogens including bacteria, viruses, or fungi and then digesting them using a combination of onboard mechanical and artificial enzymatic systems. Microbivores (2-3 $\mu \mathrm{m}$ oblate-shaped nanorobots with a mouth at one end) could achieve complete clearance of even the most severe septicemic infections in hours or less. This is far better than the weeks or months needed for antibioticassisted natural phagocytic defenses. Microbivores don't increase the risk of sepsis or septic shock because the pathogens are completely digested into harmless sugars, amino acids and the like, which are the only effluents from the nanorobot. The biocompatibility ${ }^{31 a}$ (including immunoreactivity, ${ }^{31 \mathrm{~b}}$ thrombogenicity, ${ }^{31 \mathrm{c}}$ phagocytosis ${ }^{31 \mathrm{~d}}$ and granulomatous reaction ${ }^{31 \mathrm{e}}$ ) of diamondoid medical 
nanorobots such as respirocytes and microbivores has been extensively reviewed in a book-length treatment elsewhere. ${ }^{31}$ Note that such devices will likely require some form of biocompatible coating ${ }^{31 \mathrm{f}}$ (depending on mission type and duration) to give them a high level of biocompatibility closer to that of lipid-based systems ${ }^{32}$ than to that of uncoated fullerene- or carbon nanotube-based systems. ${ }^{33}$

\section{PHARMACYTES}

The exemplar pharmacyte would not be a relatively passive nanoparticle but rather would be a medical nanorobot $1-2 \mu \mathrm{m}$ in size. It would be capable of carrying up to $\sim 1$ $\mu \mathrm{m}^{3}$ of pharmaceutical payload stored in onboard tanks that are mechanically offloaded using molecular sorting pumps $^{1 \mathrm{1a}, 21}$ mounted in the hull, operated under the control of an onboard computer. Depending on mission requirements, the payload can be discharged into the proximate extracellular fluid ${ }^{1 \mathrm{~s}}$ or delivered directly into the cytosol using a transmembrane injector mechanism. ${ }^{1 p-1 \mathrm{r}, 31 \mathrm{k}}$ The sorting pumps are typically envisioned as $\sim 1000 \mathrm{~nm}^{3}$-size devices that can transfer $\sim 10^{6}$ molecules $/ \mathrm{sec}^{1 \mathrm{la}}$ Each pump employs reversible binding sites mounted on a rotating structure that cycles between the interior and exterior of the nanorobot, allowing transport of a specific molecule even against a considerable concentration gradient. Other reversible binding sites comprise sensors on the surface of the nanorobot in order to recognize the unique biochemical signature of specific vascular and cellular addresses, ${ }^{\text {b }}$ simultaneously testing encountered biological surfaces for a sufficiently reliable combination (at least 5-10 in number) of positive-pass and negative-pass molecular markers to ensure virtually $100 \%$ targeting accuracy. Onboard power may be provided by glucose and oxygen drawn from the local environment (e.g., circulating blood, interstitial fluid, or cytosol) that is metabolized using fuel cells ${ }^{1 \mathrm{c}}$ or other methods ${ }^{1 \mathrm{~d}}$ of biochemical energy conversion. If needed for a particular application, deployable mechanical cilia ${ }^{1 \mathrm{e}}$ and other locomotive systems ${ }^{1 \mathrm{f}}$ can be added to the pharmacyte to permit transvascular ${ }^{1 \mathrm{~g}}$ and transcellular ${ }^{\text {lh }}$ mobility, thus allowing delivery of pharmaceutical molecules to specific cellular and even intracellular addresses with negligible error. Because sorting pumps can be operated reversibly, pharmacytes could also be used to selectively extract specific molecules from targeted locations as well as deposit them. Pharmacytes, once depleted of their payloads or having completed their mission, would be recovered from the patient via centrifuge nanapheresis ${ }^{1 \mathrm{i}}$ or by conventional excretory pathways. The nanorobots might then be recharged, reprogrammed and recycled for use in a second patient who may need a different pharmaceutical agent targeted to different tissues or cells than in the first patient.

Phagocytosis ${ }^{31 \mathrm{~d}}$ and foreign-body granulomatous reaction $^{31 e}$ are major issues for all medical nanorobots intended to remain in the body for extended durations, though short-duration pharmacytes that can quickly be extracted from the body ${ }^{1 i}$ may face somewhat fewer difficulties. In either case, bloodborne 1-2 $\mu \mathrm{m}$ pharmacytes can avoid clearance by the RES (whether via geometrical trapping $^{31 g}$ or phagocytic uptake ${ }^{31 \mathrm{~h}}$ ) and techniques have been proposed for phagocyte avoidance and escape ${ }^{31 i}$ at each step in the phagocytic process. Pharmacytes will not embolize small blood vessels because the minimum viable human capillary that allows passage of intact erythrocytes and white cells is 3-4 $\mu \mathrm{m}$ in diameter, which is larger than the largest proposed pharmacyte. Pharmacytes can also be equipped with mobility systems ${ }^{1 \mathrm{e}, \text { if }}$ to allow mechanically-assisted passage through partially occluded vessels or unusually narrow spaces such as the interendothelial slits of the spleen. ${ }^{31 j}$ Targeting ligands or receptors in the cell membrane exterior can be recognized by chemotactic sensors ${ }^{1 t}$ on the nanorobot surface, but note that the pharmacyte (as distinguished from conventional nanoparticles) need not always be endocytosed. For example, in some cases nanorobots may use transmembrane mechanical nanoinjectors ${ }^{31 \mathrm{k}}$ to avoid having to enter a target cell. Alternatively, if the mission requires cytopenetration then endocytosis of the nanorobot may be stimulated using biomimetic ${ }^{\mathrm{lu}}$ or completely artificial $^{\text {th }}$ (including powered mechanical) methods; after payload delivery, indigestible diamondoid nanorobots will require exocytosis by similar means. Nanorobot volume is only $1-10 \mu \mathrm{m}^{3}$ compared to $10^{3}-10^{4} \mu \mathrm{m}^{3}$ for most human tissue cells so pharmacytes could be targeted to an intracellular organelle, though nanorobots would have insufficient room to enter one (excepting perhaps the ER and nucleus) and would have to rely on nanoinjection in those cases.

There are many potential uses of pharmacytes but it will suffice to briefly mention just two general classes of applications.

First, it is often desired to deliver cytocidal agents to tumor cells. Current methods involve introducing large quantities of chemotherapy agents into the body in an effort to kill a relatively few cancerous cells, with numerous unwanted side effects on healthy cells. Precise targeting using pharmacytes can ensure delivery only to the correct cellular addresses, with presentation of cytocidal chemical agents literally on a cell-by-cell basis. In one trivial scenario, the targeted killing of 1 billion $\left(10^{9}\right)$ cancer cells with each cell capable of being killed by $\sim 10^{6}$ precisely-delivered $\sim 1000$-dalton cytocidal molecules (i.e., lethality similar to bufagin toxin) would require a total whole-body treatment dose of just $\sim 10^{15}$ cytocidal molecules or $\sim 0.001 \mathrm{~mm}^{3}(\sim 2 \mu \mathrm{g})$ of delivered material. This dose could be carried and dispensed by one trillion pharmacyte nanorobots (total injected volume of therapeutic nanorobots $\sim 2 \mathrm{~cm}^{3}$ ) assuming that only $0.1 \%$ of the nanorobots encounter an acceptable target and are 
allowed to release a $0.001 \mu \mathrm{m}^{3}$ cytocidal payload into the targeted cell, while the remaining $99.9 \%$ of the nanorobots release nothing. After initiating cell death, unmetabolized free cytocidal molecules can be locally reacquired by the pharmacyte and subsequently transported out of the patient, thus minimizing any post-treatment collateral damage. Note that the strict size requirements for macromolecules to reach the leaky vasculature of a tumor and convectively enter its pores ${ }^{34}$ may apply to passivelydiffusing payload molecules which might be conveyed and released by pharmacytes, but these limits do not apply to the motorized active nanorobots themselves. Upon arriving in the vicinity of a tumor, the pharmacyte may deliver its payload either via direct nanoinjection ${ }^{31 k}$ (for tumor cells adjoining the vasculature) or by progressive cytopenetration $^{\text {1h }}$ through adjacent cells until the targeted tumor cell that awaits payload delivery is reached.

It is well-known that apoptotic cellular "death receptors" can be expressed on both normal and cancerous cells in the human body, so one challenge for conventional drugbased therapy is to find some way to activate death receptors selectively on cancer cells only. ${ }^{35}$ With pharmacytes, such selectivity should be simple and routine using multiple chemosensors, ${ }^{1 \mathrm{j}}$ a benefit that may be characteristic of most future nanorobot-based therapeutics. For example, if caspase cascade amplification is sufficient to permit singlesite activation of the cascade, then in principle an extracellular nanorobot intending cytocide of a detected cancerous cell could press onto the outer surface of the target cell an appropriate ligand display tool. This tool might contain suitably exposed trimeric CD95L (aka. FasL) ligand (binds to the extracellular domains of three CD95 death receptors), TNF or lymphotoxin alpha (binds to CD120a), Apo3L ligand aka TWEAK (binds to DR3), or Apo2L ligand aka TRAIL (binds to DR4 and DR5). ${ }^{35,36}$ The binding event would then activate a single death receptor complex, potentially triggering the entire irreversible cytocidal cascade. If necessary, multiple such display tools could be employed. This technique avoids much of the storage requirement for bulky consumables aboard the medical nanorobot. As another approach, molecular sorting pumps on the pharmacyte surface could be used to selectively extract from the cytoplasm of a target cell specific crucial molecular species of IAPs (Inhibitors of Apoptosis) that normally hold the apoptotic process in check. Examples include survivin, commonly found in human cancer cells, ${ }^{37}$ the transcription factor NF- $\kappa \mathrm{B}$, and Akt, which delivers a survival signal that inhibits the apoptosis induced by growth factor withdrawal in neurons, fibroblasts, and lymphoid cells. Conversely, decoy receptors (DcRs) ${ }^{38}$ that compete with DR4 and DR5 for binding to Apo2L could be saturated with intrinsically harmless but precisely engineered intracellular "chaff" ligands. With IAPs removed or DcRs blockaded, apoptosis may be free to proceed.

Pharmacytes could also tag target cells with biochemical substances capable of triggering a reaction by the body's natural defensive or scavenging systems, a strategy called "phagocytic flagging". ${ }^{\mathrm{k}}$ For example, novel recognition molecules are expressed on the surface of apoptotic cells. In the case of $\mathrm{T}$ lymphocytes, one such molecule is phosphatidylserine, a lipid that is normally restricted to the inner side of the plasma membrane ${ }^{1 \mathrm{~m}}$ but, after the induction of apoptosis, appears on the outside. ${ }^{39}$ Cells bearing this molecule on their surface can then be recognized and removed by phagocytic cells. Seeding the outer wall of a target cell with phosphatidylserine or other molecules with similar action could activate phagocytic behavior by macrophages, which had mistakenly identified the target cell as apoptotic. Loading the target cell membrane surface with B7 costimulator molecules also permits T-cell recognition, allowing an immunologic response via the immunological synapse. ${ }^{40}$ These tagging operations should work well against cells that have an apoptotic response that can be triggered by cytotoxic $\mathrm{T}$ cells- such as human cancer cells and cysts.

A second major application area of pharmacytes would be the control of cell signaling processes. As a trivial example, $\mathrm{Ca}^{++}$serves as an intracellular mediator in a wide variety of cell responses including secretion, cell proliferation, neurotransmission, cellular metabolism (when complexed to calmodulin), and signal cascade events that are regulated by calcium-calmodulin-dependent protein kinases and adenylate cyclases. The concentration of free $\mathrm{Ca}^{++}$in the extracellular fluid or in the cell's internal calcium sequestering compartment (which is loaded with a binding protein called calsequestrin) is $\sim 10^{-3}$ ions $/ \mathrm{nm}^{3}$. However, in the cytosol, free $\mathrm{Ca}^{++}$concentration varies from $6 \times 10^{-8}$ ions $/ \mathrm{nm}^{3}$ for a resting cell up to $3 \times 10^{-6}$ ions $/ \mathrm{nm}^{3}$ when the cell is activated by an extracellular signal; cytosolic levels $>10^{-5}$ ions $/ \mathrm{nm}^{3}$ may be toxic, e.g., via apoptosis. To transmit an artificial $\mathrm{Ca}^{++}$activation signal to a typical $(20 \mu \mathrm{m})^{3}$ tissue cell in $\sim 1 \mathrm{msec}$, a single pharmacyte stationed in the cytoplasm must promptly raise the cytosolic ion count from $480,000 \mathrm{Ca}^{++}$ions to 24 million $\mathrm{Ca}^{++}$ions. This is a transfer rate of $\sim 2.4 \times 10^{10} \mathrm{ions} / \mathrm{sec}$ which may be accomplished using $\sim 24,000$ hull-mounted molecular sorting pumps ${ }^{1 \mathrm{a}}$ across a total nanorobot emission surface area of $\sim 2.4 \mu \mathrm{m}^{2}$. Onboard storage volume of $\sim 1 \mu \mathrm{m}^{3}$ can hold up to $\sim 20$ billion calcium atoms, enough to transmit up to $\sim 1000$ artificial $\mathrm{Ca}^{++}$signals into the cell even assuming no reabsorption and recycling of the ions.

Properly configured in cyto pharmacytes could also modify natural intracellular message traffic according to preprogrammed rules or by following external commands issued by the supervising physician. In the case of steroids and thyroid hormones, this may involve the direct manipulation of the signaling molecules themselves (after they have passed through the cell membrane) or their bound receptor complexes. However, most signaling molecules are absorbed at the cell surface, initiating a 
signal cascade which may be modulated by manipulating second-messenger molecules or other components of the in cyto signal cascade. A few basic examples of signal modifying action involving cAMP would include:

(A) Amplification. A single epinephrine molecule received by a beta adrenergic receptor at a cell surface transduces the activation of dozens of G-protein alpha subunits, each of which in turn activates a single adenylate cyclase enzyme which cyclizes hundreds of ATP molecules into cAMP molecules. The intracellular population of cAMP (in muscle or liver target cells) is normally $<10^{-6} \mathrm{M}$ or $\sim 5$ million molecules for a typical $(20 \mu \mathrm{m})^{3}$ tissue cell. Stimulation by epinephrine raises the cAMP population to $\sim 25$ million molecules in a few seconds. However, upon detecting this rising tide of cAMP during the first few msec, each in cyto pharmacyte could quickly amplify this existing chemical signal by releasing 20 million cAMP molecules (occupying a storage volume of $\sim 0.01 \mu \mathrm{m}^{3}$ ) from onboard inventories in $\sim 1 \mathrm{msec}$ - thus accelerating cellular response time by several orders of magnitude.

(B) Suppression. Similarly, upon detection of rising cAMP levels in target cells, resident pharmacytes could use molecular pumps to rapidly remove cAMP from the cytosol as quickly as it is formed, even under maximum adrenal stimulation. The diffusion-limited intake current at the basal concentration $\left(\sim 6 \times 10^{-7}\right.$ molecules $\left./ \mathrm{nm}^{3}\right)$ for a cAMP-absorbing spherical nanodevice $1 \mu \mathrm{m}$ in radius is $\sim 4$ million molecules $/ \mathrm{sec},{ }^{1 \mathrm{n}}$ so a single such device could probably keep up with natural cAMP production rates and thus completely extinguish the response by preserving a flat basal concentration even in the face of a maximum stimulus. (As a practical matter, it may be more efficient to control epinephrine generation at its glandular source unless it is desired to interface with just a single tissue type.) Simultaneously, the cAMP-absorbing nanorobot may hydrolyze the stored cAMP in the manner of the cAMP phosphodiesterases, then excrete these deactivated AMP messenger molecules back into the cytosol. Similar methods might be useful in ligand-gated ion channel desensitization or in disease symptom suppression-as, for example, in suppressing the prolonged elevation of cAMP in intestinal epithelial cells associated with the cholera toxin, that produces severe diarrhea by causing a large influx of water into the gut.

(C) Replacement. Combining suppression and amplification, an existing chemical signal could be eliminated and replaced by a different—even an opposite-message pathway using pharmacyte mediators. Alternative pathways may be natural or wholly synthetic. Novel responses to existing signals may be established within the cell to enhance functionality or to improve stability or controllability. For instance, detection of one species of cytokine by a pharmacyte could trigger rapid specific absorption of that cytokine and a simultaneous fast release of another (different) species of cytokine in its place. Such procedures must of course take into account the many redundant signaling pathways and backup systems (e.g., developmental signals, immune system, blood clotting) that exist within the cell. Medical nanorobots can allow the replacement of many redundant pathways with more refined and specific responses.

(D) Linkage. Previously unlinked signal cascades may be artificially linked using in cyto nanorobots. As a fanciful example, the receipt of epinephrine by pharmacytes located in the capillaries of the brain could trigger these devices to suppress the adrenalin response while simultaneously releasing chemical messengers producing message cascades that stimulate production of enkephalins or other opioids, thus encouraging a subjective state of psychological relaxation rather than the "fight or flight" response to certain stressful conditions.

\section{References and Notes}

1. R. A. Freitas, Jr., Nanomedicine, Volume I: Basic Capabilities, Landes Bioscience, Georgetown, TX (1999); Sections (a) 3.4.2, (b) 8.5.2.2, (c) 6.3.4.5, (d) 6.3.4, (e) 9.3.1, (f) 9.4, (g) 9.4.4.1, (h) 9.4.5, (i) 10.3.6, (j) 4.2, (k) 10.4.1.2, (m) 8.5.3.2, (n) 3.2.2, (o) 8.2.1.2, (p) 9.2.4, (q) 9.2.5, (r) 10.4.2.1, (s) 9.2.6, (t) 4.2.8, (u) 9.4.5.4; http://www.nanomedicine.com/NMI.htm.

2. R. A. Freitas, Jr., Nanomedicine: Nanotech. Biol. Med. 1, 2 (2005).

3. R. A. Freitas, Jr., J. Comput. Theor. Nanosci. 2, 1 (2005).

4. W. Tang, H. Xu, R. Kopelman, and M. A. Philbert, Photochem. Photobiol. 81, 242 (2005).

5. X. Shi, I. J. Majoros, and J. R. Baker, Jr., Mol. Pharm. 2, 278 (2005).

6. "Tecto-Dendrimers," Michigan Nanotechnology Institute for Medicine and Biological Sciences (2005); http://nano.med./edu. projects/tecto-dendrimers.htm.

7. K. Ishiyama, M. Sendoh, and K. I. Arai, J. Magnetism Magnetic Mater. 242-245, 1163 (2002).

8. J. B. Mathieu, S. Martel, L. Yahia, G. Soulez, and G. Beaudoin, Biomed. Mater. Eng. 15, 367 (2005)

9. K. B. Yesin, P. Exner, K. Vollmers, and B. J. Nelson, in 8th International Conference on Medical Image Computing and Computer Assisted Intervention (MICCAI), p. 819 (2005).

10. D. D. Chrusch, B. W. Podaima, and R. Gordon, in Conf. Proc. 2002 IEEE Canadian Conference on Electrical and Computer Engineering, edited by W. Kinsner and A. Sebak, IEEE (2002).

11. K. Hamad-Schifferli, J. J. Schwartz, A. T. Santos, S. Zhang, and J. M. Jacobson, Nature 415, 152 (2002).

12. R. F. Service, Science 298, 2322 (2002).

13. T. R. Kelly, H. De Silva, and R. A. Silva, Nature 401, 150 (1999).

14. T. J. Huang, W. Lu, H.-R. Tseng, B. Brough, A. Flood, B.-D. Yu, J. F. Stoddart, and C.-M. Ho, 11th Foresight Conf. Mol. Nanotech. (2003).

15. D. A. Leigh, J. K. Y. Wong, F. Dehez, and F. Zerbetto, Nature 424, 174 (2003).

16. N. Koumura, R. W. Zijlstra, R. A. van Delden, N. Harada, and B. L. Feringa, Nature 401, 152 (1999).

17. J. Cumings and A. Zettl, Science 289, 602 (2000).

18. A. M. Fennimore, T. D. Yuzvinsky, W.-Q. Han, M. S. Fuhrer, J. Cumings and A. Zettl, Nature 424, 408 (2003).

19. Y. Shirai, A. J. Osgood, Y. Zhao, K. F. Kelly, and J. M. Tour, Nano Lett. 5 (2005).

20. "Rice Scientists Build World's First Single-molecule Car" (2005) http://www.sciencedaily.com/releases/2005/10/051021021040.htm.

21. K. E. Drexler, "Nanosystems: Molecular Machinery, Manufacturing, and Computation," John Wiley \& Sons, New York (1992). 
22. R. C. Merkle and R. A. Freitas, Jr., J. Nanosci. Nanotechnol. 3, 319 (2003).

23. N. Oyabu, O. Custance, I. Yi, Y. Sugawara, and S. Morita, Phys. Rev. Lett. 90, 176102 (2003).

24. R. A. Freitas, Jr., A Simple Tool for Positional Diamond Mechanosynthesis, and its Method of Manufacture, U.S. Provisional Patent Appl. No. 60/543,802, U. S. Patent Pending (2005).

25. R. A. Freitas, Jr. and R. C. Merkle, Kinematic Self-Replicating Machines, Landes Bioscience, Georgetown, TX (2004); http://www. molecularassembler.com/KSRM.htm.

26. P. Vettiger, G. Cross, M. Despont, U. Drechsler, U. Duerig, B. Gotsmann, W. Haeberle, M. Lantz, H. Rothuizen, R. Stutz, and G. Binnig, IEEE Trans. Nanotechnol. 1, 39 (2002).

27. D. Bullen, S. Chung, X. Wang, J. Zou, C. Liu, and C. Mirkin, Proc. Mat. Res. Soc. 758, LL4.2.1 (2002).

28. S. Martel and I. Hunter, Proceedings of the 3rd International Workshop on Microfactories IWMF'02, Carnegie Mellon Univ. Robotics Institute (2002), p. 97.

29. R. A. Freitas, Jr., Artif. Cells Blood Subst. Immobil. Biotech. 26, 411 (1998).

30. R. A. Freitas, Jr., J. Evol. Technol. 14, 1 (2005).

31. R. A. Freitas, Jr., Nanomedicine, Volume IIA: Biocompatibility, Landes Bioscience, Georgetown, TX (2003); Sections (a) 15.2, (b) 15.2.3, (c) 15.2.5, (d) 15.4.3, (e) 15.4.3.5, (f) 15.2.2, (g) 15.4.2, (h) 15.4.3.2, (i) 15.4.3.6, (j) 15.4.2.3, (k) 15.5.7.2.3; http://www.nanomedicine.com/NMIIA.htm.

32. J. M. Koziara, J. J. Oh, W. S. Akers, S. P. Ferraris, and R. J. Mumper, Pharm. Res. 22, 1821 (2005).

33. A. Radomski, P. Jurasz, D. Alonso-Escolano, M. Drews, M. Morandi, T. Malinski, and M. W. Radomski, Br. J. Pharmacol. 146, 882 (2005).

34. E. B. Brown, Y. Boucher, S. Nasser, and R. K. Jain, Microvasc. Res. 67, 231 (2004).

35. A. Ashkenazi and V. M. Dixit, Science 281, 1305 (1998).

36. G. Pan, K. O'Rourke, A. M. Chinnaiyan, R. Gentz, R. Ebner, J. Ni and V. M. Dixit, Science 276, 111 (1997).

37. G. Ambrosini, C. Adida, and D. C. Altieri, Nature Med. 3, 917 (1997).

38. J. P. Sheridan, S. A. Marsters, R. M. Pitti, A. Gurney, M. Skubatch, D. Baldwin, L. Ramakrishnan, C. L. Gray, K. Baker, W. I. Wood, A. D. Goddard, P. Godowski, and A. Ashkenazi, Science 277, 818 (1997).

39. V. A. Fadok, D. R. Voelker, P. A. Campbell, J. J. Cohen, D. L. Bratton, and P. M. Henson, J. Immunol. 148, 2207 (1992).

40. A. Grakoui, S. K. Bromley, C. Sumen, M. M. Davis, A. S. Shaw, P. M. Allen, and M. L. Dustin, Science 285, 221 (1999).

Received: 2 November 2005. Revised/Accepted: 29 November 2005. 